\title{
Towards Network-Enabled Cognition
}

\author{
Nigel Shadbolt \\ School of Electronics and Computer Science \\ University of Southampton \\ nrs@ecs.soton.ac.uk
}

\begin{abstract}
Motivation - The increasing use of network technologies and their ability to support human collaboration, coordination and cognition is a disruptive feature of modern life. Research approach-The promise of this type of Network-Enabled Cognition arises from the formulation of conceptual frameworks based on our understanding of cognition and the implementation of a wide variety of network-enabled knowledge intensive systems. Findings/Design - A set of challenges and issues arise from this view of augmented cognition. Research limitations/Implications - These challenges and issues have clear implications for the kinds of research we may need to undertake in the future. Take away message - This keynote address will describe the concept of Network-Enabled Cognition and discuss its implications for our understanding of human decision-making.
\end{abstract}

\section{Keywords}

Networks, cognition, augmented cognition, network-enabled cognition.

\section{INTRODUCTION}

The notion of 'shared understanding' and 'problem solving' in collaborative contexts is an important focus of attention in our attempt to understand the role of contemporary networks in shaping, guiding and influencing cognition (at both the individual and collective levels) (Smart, Engelbrecht, Braines, Strub, \& Hendler, 2009a). This is particularly true in the military, emergency services and increasingly many civilian contexts (Smart, Bahrami, Braines, McRae-Spencer, Yuan, \& Shadbolt, 2007). At the collective level, networks provide the means by which agents are able to communicate, exchange and manipulate information in order to coordinate their collective actions in the service of joint or common goals (Bahrami, Yuan, Smart \& Shadbolt, 2007). At the individual level, contemporary network systems are potentially poised to play a transformative role in our notions of problem solving competence.

As we move toward an era of ubiquitous and network-mediated information access, the processing loops that drive our thoughts and actions will become increasingly subject to the possibility of deep forms of integration, forms of integration that increasingly blur the boundary between cognitive agents and their wider social and technological world (Clark, 2008). The vision is one in which networks play a dual (i.e. individual vs. collective) role in shaping and augmenting cognitive processing (Hollan, Hutchins \& Kirsh, 2000).

This does not mean, that our explanatory frameworks need to be divided along similar lines. The mechanisms that shape our individual thoughts and action should align themselves, at some level of abstraction, with those that enable agent coalitions to direct and orchestrate their behaviours in the realization of common goals. At its most extreme, the claim is that the patterns of information flow and processing within a highly inter-connected community of collaborative problem-solving agents is similar to that seen in the more localized (agent-centred) versions of human cognitive processing.

In understanding the power and potential of network systems to promote mutual understanding and support of coordinated action we face a number of challenges and issues. These include the following:

1. What is the relationship between network parameters (such as topology, diffusion dynamics, etc.) and cognitive performance at both the individual and collective levels? Do specific types of network structure impair or improve the quality of team-based decision outcomes?

2. What is the value of network analyses in understanding the distinctive contributions of a network environment to individual and collective cognitive processing? Do such analyses shed any light on how rich ensembles of heterogeneous, but interconnected, elements are able to realize the kind of properties we equate with human intelligence?

3. How can network-accessible information content be adapted to suit the specific cognitive and perceptual processing biases of distinct agent communities? How can we improve the reliability, quality and trustworthiness of information content (Huynh, 2009), and how can we re-present information content so that it is better able to scaffold and augment our thoughts and actions? 
4. How can we reconcile the inherent tension between the potential of networks to empower cognition and the fact that networks provide new routes to social deception and social manipulation? How can we protect networkmediated forms of shared understanding and collaborative problem solving from the effects of false or poor quality information?

5. How can we optimize the flow of information in a network environment so as to better support, create and maintain shared understanding? How do changes in network structure affect our ability to ensure continued shared understanding? And how can we use networks to detect breakdowns or shortfalls in shared understanding?

6. What is the effect of using semantically-enriched representations of network-accessible information content on the shared understanding of agent coalitions?

In addition to these challenges we must also confront the possibility that network systems could impact individual and collective cognitive capabilities in profound and largely unpredictable ways (Tollefsen, 2006). Networks may do more than just enable cognition; it may be difficult for certain types of problem solving to exist without them (Hendler, Shadbolt, Hall, Berners-Lee \& Weitzner, 2008).

This keynote talk will address the challenges and opportunities that arise with this view of Network-Enabled Cognition. It will reflect on how this influences our view of methodologies, techniques and frameworks such as NDM. A significant number of the examples we draw on are based on our involvement in a number of recent and ongoing projects with the US and UK military (Smart, Liang, Booth, Russell, Briscombe, Rankin \& Shadbolt, 2009b; Rasmussen, Sieck \& Smart, In Press).

\section{ACKNOWLEDGMENTS}

Thanks to Paul Smart and colleagues for much of the inspiration and implementation of the ideas presented herein. The work was supported by grants from the joint US ARL and UK MoD International Technology Alliance Programme, the UK's MoD Defence Technology Centre in Data and Information Fusion, and the UK's Engineering and Physical Sciences Research Council.

\section{REFERENCES}

Bahrami, A., Yuan, J., Smart, P. R., \& Shadbolt, N. R. (2007) Context-Aware Information Retrieval for Enhanced Situation Awareness. Military Communications Conference (MILCOM), Orlando, Florida, USA.

Clark, A. (2008) Supersizing the Mind: Embodiment, Action, and Cognitive Extension Oxford University Press, USA.

Hendler, J., Shadbolt, N. R., Hall, W., Berners-Lee, T., \& Weitzner, D. (2008) Web Science: An Interdisciplinary Approach to Understanding the Web. Communications of the ACM, 51(7), 60-69.

Hollan, J., Hutchins, E., \& Kirsh, D. (2000) Distributed cognition: toward a new foundation for human-computer interaction research. ACM Transactions on Computer-Human Interaction (TOCHI), 7(2), 174-196.

Huynh, T. D. (2009) A Personalized Framework for Trust Assessment. ACM Symposium on Applied Computing Trust, Reputation, Evidence and other Collaboration Know-how (TRECK) Track, Honolulu, Hawaii, USA.

Rasmussen, L. J., Sieck, W. R., \& Smart, P. R. (in press) Cultural Variations in Mental Models of Planning and Their Implications for Supporting Multinational Collaboration. Journal of Cognitive Engineering and Decision Making.

Smart, P. R., Bahrami, A., Braines, D., McRae-Spencer, D., Yuan, J., \& Shadbolt, N. R. (2007) Semantic Technologies and Enhanced Situation Awareness. 1st Annual Conference of the International Technology Alliance (ACITA'07), Maryland, USA.

Smart, P. R., Engelbrecht, P., Braines, D., Strub, M., \& Hendler, J. (2009a) Cognitive Extension and the Web. Web Science Conference: Society On-Line, Athens, Greece.

Smart, P. R., Liang, S. F., Booth, C., Russell, A., Briscombe, N., Rankin, A., \& Shadbolt, N. R. (2009b) Using Semantic Technologies to Improve Information Exploitation in Military and Civilian Application Contexts. Knowledge Systems for Coalition Operations (KSCO'09), Southampton, UK.

Tollefsen, D. P. (2006) From extended mind to collective mind. Cognitive Systems Research, 7(2-3), 140-150. 